\title{
Spontaneous knee dislocation subsequent to intramedullary nailing of femoral shaft fracture
}

\begin{abstract}
Knee dislocation is a rare and devastating injury, may cause limb-threatening sequelae if damage to nerves and blood vessels. We describe a case of femoral shaft fracture without initial knee dislocation followed by stabilization with intramedullary nailing. When the patient postoperatively regained consciousness from anesthesia, he had restless agitation that caused an obvious knee dislocation. Emergency physicians need to keep in mind that spontaneous dislocation or relocation of the knee can occur at any point in time after trauma.
\end{abstract}

Keywords: Knee dislocation, Femoral shaft fracture, Intramedullary nailing, Agitation
Volume I Issue 2 - 2014

\section{Chun-Yu Chen, Kuan-Yu Lin, Yih-Wen Tarng, Jenn-Huei Renn, Kai-Cheng Lin}

Department of Orthopedics, Kaohsiung Veterans General Hospital,Taiwan

Correspondence: Kai-Cheng Lin, Department of

Orthopaedics, Kaohsiung Veterans General Hospital, 386

Ta-Chung Ist Road, Kaohsiung, Taiwan, Tel +886-7-3422 I 2 I ext 305।, Fax+886-7-3420478, Email orthokcl@gmail.com

\section{Abbreviations: ED, Emergency Department \\ Introduction}

Knee dislocations are rarely observed with high-energy trauma, but they usually present potential for neurovascular damage and complex ligament lesions. We describe a case of femoral shaft fracture presented at our emergency department (ED). When the patient postoperatively regained consciousness from anesthesia, he had restless agitation that caused an obvious knee dislocation. It is important for the emergency physician to keep in mind that in some cases high energy is not entirely dissipated by femoral shaft fracture; it may also injure the complex ligaments of the knee joint, resulting in subsequent knee dislocation for various reasons. And we obtained the patient's informed written consent for print and electronic publication of the case report.

\section{Case presentation}

A 37-year-old man arrived at our ED with a painful left thigh deformity following a motor vehicle accident. On examination, the pulsation and skin color were intact. X-ray of the femur revealed a transverse fracture with comminuted fragments of the middle shaft (Figure 1). He underwent closed reduction and internal fixation with an intramedullary nail for left femoral shaft fracture on the same day. Postoperative X-ray revealed appropriate reduction and nail position as well as normal knee joint alignment (Figure 2). When the patient regained consciousness in the recovery room, his restless agitation caused knee dislocation, resulting in a deformity (Figure 3). The dislocation was reduced with traction under emergent intravenous sedation. Upon re-evaluation of the injured extremity, pulsation as well as skin color and temperature were normal. CT and MRI scans revealed a tear in the anterior and posterior cruciate ligaments and the posterolateral corner complex.

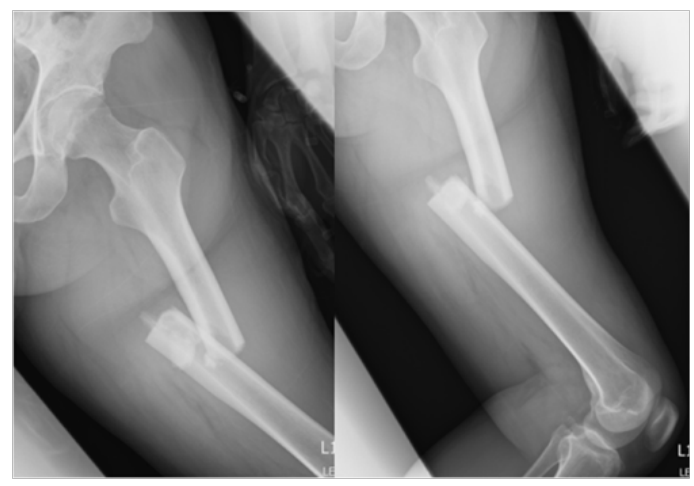

Figure I Transverse fracture with comminuted fragments of the femoral shaft.

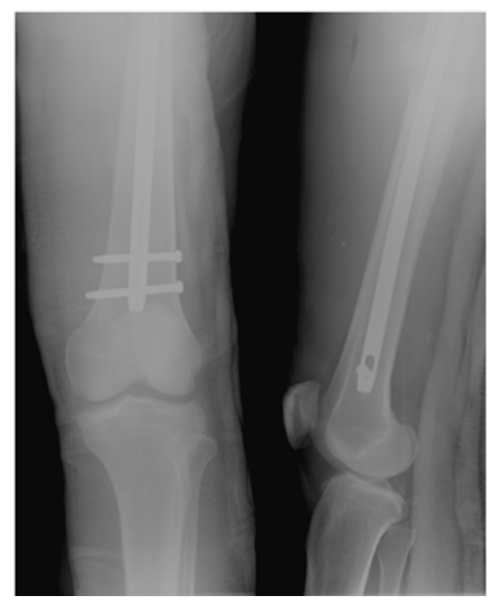

Figure 2 Appropriate reduction and nail position with normal knee joint alignment. 


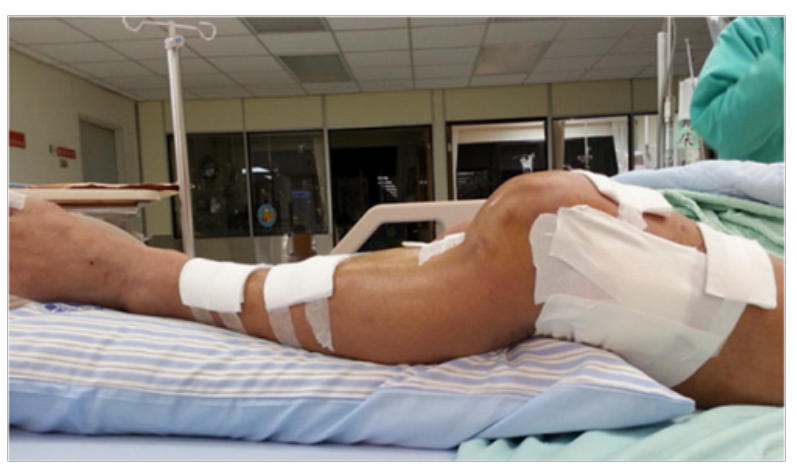

Figure 3 Restless agitation caused knee dislocated deformity.

\section{Discussion}

Isolated femoral shaft fractures are commonly associated with high-energy trauma, requiring evaluation of both the hip and knee for associated injuries. Concurrent damage to the knee may involve only ligament injury, ${ }^{1}$ however, the damage may be severe enough to cause knee dislocation. ${ }^{2,3}$ The patient usually presents a lower degree of pain and swelling around the knee than the thigh. Unless there is obvious knee deformity because of dislocation, the clinician may overlook knee ligament injury when viewing normal knee radiographs. Knee dislocation is a rare and devastating injury resulting from either high- or low-energy trauma. High-energy trauma is commonly associated with a dashboard injury, thereby resulting in axial load on the flexed knee. Low-energy trauma often results from athletic injury or morbid obesity. ${ }^{4}$ Types of ligament injuries vary; immediate reduction and careful evaluation of neurovascular status have been suggested. Damage to nerves and blood vessels may cause limb-threatening sequelae if neglected. Twaddle et al..$^{5}$ Prospectively evaluated soft tissue injury patterns in 60 patients with 63 dislocatable knees and reported the incidence of popliteal artery injury $(14 \%)$ and peroneal nerve palsy (14\%). Because of the occurrence of spontaneous reduction before arrival at the ED, the incidence of knee dislocations is commonly underestimated in $50 \%$ cases. ${ }^{6}$ During these scenarios, clinicians may fail to evaluate neurovascular injury because of no obvious evidence of knee dislocation in radiographs or gross views. Performing an angiography in the presence of clinically palpable pulses is debatable. Martinez et al. ${ }^{7}$ reported that in a series of 12 patients with normal peripheral pulses, only 1 had an intimal defect, and none of these patients required surgical intervention. Hence, a good peripheral pulse has been considered as a reliable sign of adequate circulation. Current evidence supports the view that angiography is not essential if the peripheral pulse is normal. A Doppler flow examination can adequately exclude the possibility of vascular injury. ${ }^{8}$

The type of complex injury as in our case is rarely reported..$^{2,3} \mathrm{~A}$ literature review indicates that this is the first reported case without initial knee dislocation followed by stabilization with intramedullary nailing of the femoral fracture under intact neurovascular status, but spontaneous knee dislocation occurred when regaining consciousness with restless agitation in the recovery room. To our knowledge, transverse or comminuted shaft fractures are usually due to direct contusion instead of spiral torsion. The energy is usually dissipated over the fracture site without transmission to the soft tissue of the upper and lower joints. Therefore, cases with multiple knee ligament injuries leading to dislocation are rare. After carefully evaluating the mechanism of trauma in this patient, we observed that his lower leg was trapped under the motorcycle, after which his thigh collided directly with a solid object. The torque energy may have occurred because the lower leg was trapped.

\section{Conclusion}

This case provides emergency and orthopedic physicians some important perspectives. First, spontaneous dislocation or relocation of the knee can occur at any point in time; frequent and repeated checking of the peripheral pulse is very important. Second, the fracture pattern does not reveal the complete trauma mechanism. There may be more complex mechanisms that should be carefully evaluated by obtaining complete information from the patient.

\section{Acknowledgments}

None.

\section{Conflicts of interest}

The author declares that there is no conflict of interest.

\section{References}

1. Moore TM, Patzakis MJ, Harvey JP Jr. Ipsilateral diaphyseal femur fractures and knee ligament injuries. Clin Orthop Relat Res. 1988;(232):182-189.

2. Matthews DE, Jones GS, Hughes JL. Femur fracture with ipsilateral knee dislocation. Orthopedics. 1993;16(8):917-919.

3. Giannoudis PV, Roberts CS, Parikh AR, et al. Knee dislocation with ipsilateral femoral shaft fracture: a report of five cases. J Orthop Trauma. 2005;19(3):205-210.

4. Folt J, Vohra T. Low-velocity knee dislocation in the morbidly obese. Am J Emerg Med. 2012;30(9):e5-e6.

5. Twaddle BC, Bidwell TA, Chapman JR. Knee dislocations: where are the lesions? A prospective evaluation of surgical findings in 63 cases. J Orthop Trauma. 2003;17(3):198-202.

6. Wascher DC. High-velocity knee dislocation with vascular injury. Treatment principles. Clin Sports Med. 2000;19(3):457-477.

7. Martinez D, Sweatman K, Thompson EC. Popliteal artery injury associated with knee dislocations. Am Surg. 2001;67(2):165-167.

8. Chen CE, Wang JW. Floating knee with ipsilateral knee dislocation: case report. J Trauma. 1999;44(4):735-737. 